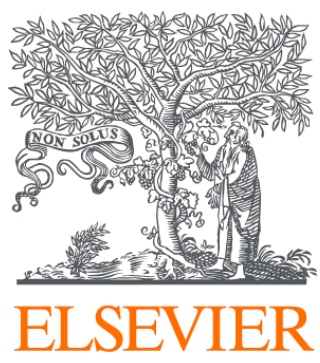

Since January 2020 Elsevier has created a COVID-19 resource centre with free information in English and Mandarin on the novel coronavirus COVID-

19. The COVID-19 resource centre is hosted on Elsevier Connect, the company's public news and information website.

Elsevier hereby grants permission to make all its COVID-19-related research that is available on the COVID-19 resource centre - including this research content - immediately available in PubMed Central and other publicly funded repositories, such as the WHO COVID database with rights for unrestricted research re-use and analyses in any form or by any means with acknowledgement of the original source. These permissions are granted for free by Elsevier for as long as the COVID-19 resource centre remains active. 


\title{
Virologie : l'apport de la biologie moléculaire dans le diagnostic microbiologique en pneumopédiatrie
}

\section{Microbiological approaches with molecular biological diagnosis to paediatric respiratory viral infections}

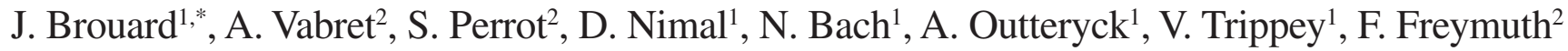 \\ ${ }^{1}$ Service de pédiatrie, avenue Clémenceau, CHU de Caen, 14033 Caen cedex, France \\ ${ }^{2}$ Laboratoire de virologie humaine et moléculaire, CHU de Caen, avenue Clémenceau, 14033 Caen cedex, France
}

\begin{abstract}
Résumé
Les outils traditionnels du diagnostic virologique comprennent la recherche antigénique directe par immunofluorescence (IF) ou test immunoenzymatique (EIA), et l'isolement en culture. Les techniques d'IF ou EIA ont une sensibilité en général légèrement inférieure à celle de la culture, mais elles permettent de détecter également une fraction d'échantillons faussement négatifs en culture. Les résultats de plusieurs équipes, utilisant des techniques de reverse transcription-polymerase chain reaction (RT-PCR), montrent que les méthodes moléculaires détectent plus d'échantillons positifs que les méthodes traditionnelles. Le travail est en cours pour élargir le nombre de virus identifiés par des RT-PCR multiplex et découvrir de nouveaux virus, tels le métapneumovirus humain, en cause dans les atteintes respiratoires de l'enfant. En conclusion, afin de concilier rapidité, contrôle des coûts et un fort taux de détection des virus respiratoires, l'utilisation combinée de l'IF et de la RT-PCR multiplex semble ce jour la meilleure façon de progresser dans le diagnostic des maladies respiratoires de l'enfant.

(c) 2007 Elsevier Masson SAS. Tous droits réservés.
\end{abstract}

\section{Summary}

The conventionnal tools used for virological diagnosis include direct antigen detection by immunofluorescence (IFA) or an immunoenzymatic test (EIA), and viral isolation technique (VIT). In most cases, IFA and EIA have a slightly lower sensitivity than VIT but are also able to detect some VIT-negative samples. Results of several teams using RT-PCR technologies show that the molecular methods detect more positive cases than the conventional tools. Work is under way to expand the number of viruses detected by multiplex RT-PCR and to determine wether newly discovered viruses, such as human metapneumovirus, contribute to burden of paediatric lower respiratory infections. In conclusion, according to requirements of speed, low cost of the methods, and to achieve the highest rate of detection of respiratory viruses, the combined use of IFA and multiplex RT-PCR is today likely to be the best way to improve diagnosis of respiratory illnesses in children. C 2007 Elsevier Masson SAS. Tous droits réservés.

Mots-clés : Diagnostic biologique ; Infections respiratoires ; Maladies virales ; Reverse transcription-polymerase chain reaction

Les infections respiratoires virales sont une pathologie majeure chez les jeunes enfants, et une cause fréquente d'hospitalisation. Leur fréquence, leur caractère épidémique, leur gravité potentielle et leurs conséquences économiques en font un problème de santé publique.
Historiquement, les premières techniques de mise en évidence des virus respiratoires étaient basées sur l'isolement en culture. Puis, le développement des méthodes antigéniques a permis d'obtenir des résultats plus rapidement et plus facilement. Ces deux méthodes servent encore aujourd'hui de référence. Les

\footnotetext{
*Auteur correspondant.

Adresse e-mail : brouard-j@chu-caen.fr (J. Brouard).
} 
techniques de biologie moléculaire (amplification génique par polymerase chain reaction $\mathrm{PCR}$ ) prennent une place de plus en plus grande dans le diagnostic. Le choix de la méthode dépend de nombreux facteurs : type de pathologie, virus recherché, urgence du diagnostic, disponibilité des techniques au laboratoire. Les techniques de biologie moléculaire, outil plus récent, augmentent la sensibilité de la détection, mais leur utilisation en pratique de routine est discutée.

Le diagnostic étiologique de l'infection virale peut poursuivre plusieurs buts. Le plus souvent, dans le contexte de la pneumopédiatrie, il s'agit d'apporter la preuve de l'étiologie virale d'une pathologie suspectée cliniquement. L'identification précise du pathogène viral est importante : elle peut modifier le choix du traitement, sa durée, les modalités de surveillance. Elle détermine également l'arrêt d'investigations invasives qui sont entreprises habituellement dans le cadre de la recherche infectieuse.

\section{Modalités de prélèvements}

En dehors de la sérologie et de la détection d'antigènes plasmatiques où un simple sérum est nécessaire, les autres prélèvements pour analyse virologique doivent répondre à une démarche qualitative extrêmement rigoureuse. Lorsqu'un diagnostic direct est envisagé, le caractère intracellulaire obligatoire des virus impose des prélèvements contenant des cellules. La fragilité de la plupart des virus impose le recours à des milieux de transports spécifiques quand une technique de culture est envisagée. Pour parvenir au diagnostic, la qualité du prélèvement est primordiale. De même, la rapidité de la dégradation des acides nucléiques viraux rend nécessaire un acheminement et une prise en charge technique rapide des prélèvements au laboratoire, surtout quand des techniques quantitatives ou semi-quantitatives sont envisagées. Plusieurs erreurs sont couramment rencontrées. Elles sont parfois « irréversibles » lorsqu'il s'agit de prélèvements difficilement reproductibles : fixation de la totalité d'une biopsie en liquide de Bouin, rendant impossible les analyses virales moléculaires ou les cultures, absence de cellules sur un prélèvement nasopharyngé destiné à la recherche d'antigènes viraux, dégradation d'un prélèvement initialement de bonne qualité par retard d'acheminement au laboratoire. Néanmoins, les aspirations nasopharyngées restent la technique la plus efficiente pour permettre le diagnostic des principaux pathogènes viraux : virus respiratoire syncytial (VRS), virus grippal, virus parainfluenza, adénovirus reposant sur les techniques rapides de détection des antigènes viraux par immunofluorescence. Le rhinovirus ne bénéficie pas de la possibilité de détection rapide par les antigènes viraux, la culture virale le permet. Chez l'immunodéprimé, le recours systématique au lavage broncho-alvéolaire dans le diagnostic étiologique des infections respiratoires permet l'identification de multiples pathogènes viraux où prédominent le cytomégalovirus, les adénovirus et l'herpes simplex.

\section{Techniques d'analyse}

\subsection{Détection d'anticorps (sérologie)}

C'est une méthode diagnostique indirecte. Les techniques sérologiques ont évolué, permettant une standardisation, une automatisation et une réponse rapide à la question du statut immunitaire du patient vis-à-vis d'un virus. Cependant, elles restent peu applicables dans le domaine clinique des infections respiratoires virales en dehors des études épidémiologiques. La comparaison de la réactivité de deux sérums prélevés à une quinzaine de jour d'intervalle et analysés en parallèle affirme rétrospectivement la responsabilité d'un pathogène dans un tableau clinique.

\subsection{Détection de virus ou de composants viraux}

\subsubsection{Détection d'antigènes viraux}

La mise en évidence d'antigènes viraux présente plusieurs avantages par rapport à la détection directe du virus. Elle est beaucoup plus rapide, ne nécessite pas de manipuler un virus maintenu en état de réplication. L'immunofluorescence est une technique qui utilise un anticorps monoclonal marqué par la fluorescéine [1]. L'antigène ciblé peut être intracellulaire ou membranaire. Cette méthode permet de valider la qualité du prélèvement en vérifiant la présence de cellules respiratoires, elle fournit un résultat semi-quantitatif en un temps rapide. Le prélèvement doit être de bonne qualité et le délai d'exécution rapide pour préserver les cellules intactes. D'un coût faible, elle permet la détection simultanée de plusieurs virus, mais nécessite un lecteur entraîné. Au niveau du spectre des virus recherchés, cette technique est limitée par la nécessité de disposer des anticorps monoclonaux correspondant. Actuellement, elle permet de détecter les virus influenza A et B, le VRS, les virus parainfluenza 1, 2, 3, et 4, les adénovirus [2]. Récemment sont apparues des techniques immuno-enzymatiques, tests unitaires de diagnostic rapide permettant de détecter en moins d'une heure le VRS, les virus influenza $\mathrm{A}$ et $\mathrm{B}$ et les adénovirus [3]. Ces tests simples et économiques ne nécessitent pas d'équipement particulier et peuvent être éventuellement réalisés au lit du malade. Cette technique manque de sensibilité mais est compensée généralement par la présence de grandes quantités de virus infectieux dans les sécrétions nasales ou respiratoires dans le diagnostic de l'infection grippale ou du virus respiratoire syncytial [4]. 


\subsubsection{Cultures de virus}

C'est lors du recours à ces techniques que la qualité des prélèvements et de leur acheminement est la plus importante. C'est la culture sur lignée cellulaire qui est la plus utilisée [5]. La détection du virus en culture peut également avoir recours à une étape complémentaire de l'identification des antigènes viraux dans le milieu de culture. La détection de virus en culture cellulaire est très spécifique mais peut manquer de sensibilité. Cette perte de sensibilité peut être liée à une dégradation secondaire à un prélèvement réalisé dans de mauvaises conditions. Cette technique a été longtemps considérée comme la méthode de référence, notamment grâce à son principe d'amplification qui permet la détection de faibles quantités de virus [3]. Ses inconvénients sont la nécessité de locaux et d'équipements adaptés, une réalisation délicate, le délai de réponse (quatre à sept jours voire plus), un coût élevé, et le fait que certains virus se multiplient difficilement ou pas du tout en culture. De plus, il n'existe pas une lignée capable de révéler l'ensemble des virus : fibroblastes humains embryonnaires MRC5 (pour le VRS, les adénovirus, certains rhinovirus), lignées continues humaines Hep 2, HeLa 229 (paramyxovirus, adénovirus), lignées continues animales MDCK (influenza), MK2 ou Vero [6]. Le développement viral peut être révélé soit par visualisation sur la culture d'un effet cytopathogène (ECP), soit en reprenant les cellules de la culture par une technique d'immunomarquage ou de biologie moléculaire. Par rapport à l'immunofluorescence, la culture cellulaire permet d'isoler certaines souches de rhinovirus [6] et elle est en général plus performante pour les virus influenza et les adénovirus [3]. Toutefois, elle peut être faussement négative, à cause notamment de problèmes d'inhibiteurs de la croissance virale présents dans le prélèvement (anticorps, interférons) ou d'une contamination bactérienne des cultures.

\subsubsection{Virologie moléculaire}

Toutes les techniques de mise en évidence d'acides nucléiques viraux reposent sur le même principe : la capacité d'une séquence d'acide nucléique, $\mathrm{ADN}$ ou $\mathrm{ARN}$, à se lier avec sa séquence complémentaire (hybridation). Néanmoins, cette capacité ne peut être utilisée en diagnostic qu'après plusieurs étapes de préparation. Théoriquement, le diagnostic de n'importe quel virus, dont une partie de la séquence génétique est connue, est accessible. Ces techniques permettent une détection qualitative de l'acide nucléique viral, très hautement spécifique. Elles peuvent également être quantitatives, comme pour la détermination de la charge virale. Dans la plupart des cas, il est nécessaire d'avoir recours au processus d'amplification. Le couplage de la détection moléculaire aux techniques d'amplification permet de mettre en évidence des quantités infimes d'acides nucléiques viraux, assurant une grande sensibilité à ce type de technique. L'extrême sensibi- lité (puisqu'une seule copie d'acide nucléique est théoriquement suffisante pour déclencher l'amplification) est limitée par les étapes initiales d'extraction et de purification. Il existe un risque de contamination croisée des échantillons à tous les niveaux, notamment lors de la prise en charge technique au laboratoire de virologie (contamination par des produits amplifiés présents dans l'atmosphère). Contrairement aux cultures virales, la mise en évidence de l'acide nucléique ne permet pas d'attester de l'état infectieux du virus présent dans le prélèvement. Cet écueil peut être en partie contourné par l'amplification d'ARN messagers viraux (qui ne sont exprimés que lors des étapes de réplication), mais se heurte encore à des difficultés techniques importantes. Les reverse transcription-polymerase chain reaction (RT-PCR) multiplex permettant de détecter les virus respiratoires ont initialement été mises au point individuellement. Puis, devant le nombre toujours plus grand de virus recherchés et dans un souci de simplification, des techniques de « regroupement » de PCR ont été étudiées. Dans ce but, trois RT-PCR multiplex ont été mises au point et publiées récemment par le laboratoire de virologie de Caen [7]. Elles permettent d'emblée de détecter 13 virus : les virus influenza $\mathrm{A}$, virus influenza $\mathrm{B}$, metapneumovirus humain (hMPV) et VRS pour la multiplex 1 ; les virus parainfluenza $1,2,3$, et 4 pour la multiplex 2 ; les rhinovirus, entérovirus, virus influenza C, coronavirus OC42 et 229E pour la multiplex 3. Ce laboratoire a également développé une multiplex adénovirus [8] et une autre permettant d'identifier les nouveaux coronavirus NL63 et HKU1 [9].

\section{Comparaison des techniques}

Jusqu'aux années 1990, le diagnostic virologique moléculaire était limité à la recherche d'un virus par une seule technique. Mais une atteinte clinique précise n'est pas spécifique d'un virus, et un virus donné peut être à l'origine d'atteintes diverses de tout l'arbre respiratoire, tandis que de nouveaux virus impliqués dans les infections respiratoires sont régulièrement découverts. Initiées par Gilbert et al. en 1996 [10] et Freymuth et al. en 1997 [2], des techniques multiplex sont apparues, permettant la détection simultanée de plusieurs virus, elles ont montré des qualités de rapidité, sensibilité et spécificité, tout en permettant une réduction du coût des réactifs et de la main d'œuvre. En 1998, Osiowy et al. publient une RT-PCR multiplex permettant la détection du VRS, des virus parainfluenza 1, 2, et 3 et des adénovirus [11]. Un test standardisé a été ensuite commercialisé (Hexaplex ${ }^{\circledR}$, Prodesse Inc., Waukesha, Wis.) permettant la détection des virus respiratoires les plus fréquents (VRS A et B, virus influenza A et B, virus parainfluenza 1, 2 et 3) [12]. Il a été testé et comparé aux techniques conventionnelles par trois équipes différentes en 2001 [13-15] puis en 2004 dans une grande étude épidémiologique américaine 
[16]. Grondahl et al. ont décrit une RT-PCR multiplex détectant simultanément neuf virus [17]. Cette méthode a ensuite été évaluée par Puppe et al. en 2004 [18]. Enfin, Coiras et al. [19] ont présenté en 2003 une RT-PCR multiplex identifiant cinq virus respiratoires (VRS A et B, virus influenza A, B et C et adénovirus), qu'ils ont complétée en 2004 en y associant une seconde multiplex pour, au total, un panel de 14 virus. Ces techniques sont le plus souvent « maison », leurs spectres ne détectent ni les parainfluenza 4, virus probablement plus fréquents qu'il n'a été décrit jusqu'alors, ni les rhinovirus pathogènes très fréquents de l'arbre respiratoire supérieur et pouvant entraîner des atteintes des voies aériennes inférieures, ni le métapneumovirus (hMPV) et les coronavirus NL-63 et HKU1, virus récemment découverts.
Plus récemment, d'autres travaux étudient l'application pratique de ces techniques en diagnostic de routine, et les comparent aux techniques conventionnelles (Tableau 1). Le panel de virus recherchés est varié et se trouve parfois associé aux germes atypiques. Ces études utilisent en majorité des RTPCR multiplex [20,21]. Il a été montré que le fait d'associer les paires d'amorces ne nuisait pas à la sensibilité [7]. Les équipes utilisant une détection par hybridation soulignent son net avantage par rapport à la détection par électrophorèse en gel d'agarose. Syrmis et al. évaluent ce gain de sensibilité à un facteur 100 [22], et rappellent que cela évite de manipuler du bromure d'éthidium, produit cancérigène.

Dans l'ensemble, toutes ces études montrent les avantages de la détection par RT-PCR, notamment une sensibilité allant

Tableau 1

Synthèse des récentes études sur la comparaison des méthodes conventionnelles (MC) et des techniques de reverse transcription-polymerase chain reaction (RT-PCR) pour l'analyse virale des prélèvements respiratoires

\begin{tabular}{|c|c|c|c|c|c|c|c|c|}
\hline \multirow{2}{*}{$\begin{array}{l}\text { Année } \\
2000\end{array}$} & \multirow{2}{*}{$\begin{array}{l}\text { Auteur } \\
\text { Weigl [25] }\end{array}$} & \multirow{2}{*}{$\begin{array}{l}\begin{array}{l}\text { Nombre } \\
\text { échantillons }\end{array} \\
1281\end{array}$} & \multirow{2}{*}{$\begin{array}{l}\text { Nombre } \\
\text { virus } \\
9\end{array}$} & \multirow{2}{*}{$\begin{array}{l}\text { Virus outre VRS, } \\
\text { VI A B } \\
\text { + VPI } 1,3\end{array}$} & \multicolumn{2}{|c|}{ Technique et détection } & \multirow{2}{*}{$\begin{array}{l}\text { Taux de } \\
\text { positivité } \\
\text { RT-PCR } \\
35 \%\end{array}$} & \multirow{2}{*}{$\begin{array}{l}\text { Taux de } \\
\text { positivité MC }\end{array}$} \\
\hline & & & & & RT-PCR & Gel + ELISA & & \\
\hline & & & & $\mathrm{EV}, \mathrm{AdV}$ & & & & \\
\hline & & & & $\mathrm{CP}, \mathrm{MP}$ & & & & \\
\hline 2001 & Hindiyeh [13] & 300 & 6 & + VPI $1,2,3$ & Hexaplex & Hybridation & $50,7 \%$ & $49,3 \%$ \\
\hline 2001 & Liolios [15] & 143 & 6 & + VPI $1,2,3$ & Hexaplex & Hybridation & $11,9 \%$ & $5,6 \%$ \\
\hline 2004 & $\begin{array}{l}\text { Henrickson } \\
{[16]}\end{array}$ & 1347 & 6 & + VPI 1,2,3 & Hexaplex & Hybridation & $43 \%$ & $26 \%$ \\
\hline \multirow[t]{5}{*}{2004} & Coiras [19] & 201 & 14 & $+\mathrm{VIC}$ & RT-PCR & Gel & $60,2 \%$ & $19,9 \%$ \\
\hline & & & & VPI $1,2,3,4$ & & & & \\
\hline & & & & Pico, AdV & & & & \\
\hline & & & & $\mathrm{CP}, \mathrm{MP}$ & & & & \\
\hline & & & & OC43,229E & & & & \\
\hline \multirow[t]{3}{*}{2004} & Gruteke [27] & 178 & 10 & + VPI 1,3 & RT-PCR & Gel et PCR & $65 \%$ & \\
\hline & & & & EV, RV, AdV & & nichée & & \\
\hline & & & & $\mathrm{CP}, \mathrm{MP}$ & & & & \\
\hline \multirow[t]{2}{*}{2004} & Syrmis [22] & 598 & 7 & + VPI $1,2,3$ & RT-PCR & Hybridation & $33,7 \%$ & $29,9 \%$ \\
\hline & & & & AdV & & & & \\
\hline 2004 & Weinberg [20] & 668 & 5 & + VPI 1,3 & $\begin{array}{l}\text { RT-PCR } \\
\text { uniplex }\end{array}$ & Temps réel & $27,7 \%$ & $13,3 \%$ \\
\hline \multirow[t]{3}{*}{2005} & Rovida & 254 & 10 & + VPI 1,2,3 & RT-PCR & Gel & $37,7 \%$ & $29,9 \%$ \\
\hline & [21] & & & AdV, hMPV & uniplex & & & \\
\hline & & & & OC43, 229E & & & & \\
\hline \multirow[t]{5}{*}{2005} & Freymuth [9] & 449 & 15 & $+\mathrm{VIC}$ & RT-PCR & $\mathrm{Gel}+$ & $91,5 \%$ & $72,3 \%$ \\
\hline & & & & VPI $1,2,3,4$ & & hybridation & & \\
\hline & & & & EV, RV, AdV & & & & \\
\hline & & & & OC43, 229E & & & & \\
\hline & & & & NL63, hMPV & & & & \\
\hline
\end{tabular}

VI : virus influenza (A,B,C), VPI : parainfluenza (1,2,3,4), EV : entérovirus, RV : rhinovirus, Pico : Picornavirus, AdV : adénovirus, OC43-229E-NL63 : coronavirus (OC43,229E,NL63), hMPV : métapneumovirus, CP : Chlamydia pneumoniae, MP : Mycoplasma pneumoniae 
de 91 à $100 \%$, qui correspond à un gain marqué par rapport aux techniques conventionnelles. Les techniques moléculaires détectent la présence de séquences d'acides nucléiques, que le virus soit vivant ou non. Les RT-PCR permettent de s'affranchir des problèmes de qualité, de quantité, de transport et de conservation des échantillons, car elles ne dépendent que de la présence d'acides nucléiques viraux [22]. Toutefois, l'utilisation d'un contrôle interne reste nécessaire pour la validation des étapes techniques. Il est préférable d'utiliser un gène présent dans les cellules de l'échantillon (par exemple : gène de la GAPDH) plutôt qu'un contrôle interne ajouté par la suite car cela permet de confirmer la validité du prélèvement. La capacité à détecter les virus non réplicatifs ou présents en faible quantité peut être aussi un inconvénient [23,24]. En effet, les virus détectés par culture cellulaire sont réplicatifs et infectieux ; il y a donc peu de doutes sur leur implication. L'immunofluorescence apporte la preuve de l'atteinte du tissu respiratoire par la mise en évidence d'inclusions virales dans les cellules infectées [3]. De plus, cette technique permet une évaluation semi quantitative. Il est par contre plus difficile d'affirmer le rôle pathogène des virus détectés par les méthodes PCR. Elles détectent la présence du génome des virus dans les prélèvements, voire uniquement de fragments de ce génome, mais ne sont pas informatives quant à sa réplication et à son rôle pathogène. Toutefois, Liolios et al. rappellent que cette implication pathogène est assurée par les signes cliniques respiratoires ayant motivé la réalisation du prélèvement [15]. Grâce à cette sensibilité, la biologie moléculaire peut aussi être un outil épidémiologique pour analyser la circulation des virus dans la population générale. C'est aussi un avantage dans certaines pathologies où une faible charge virale suffit parfois à déclencher le processus inflammatoire (asthme par exemple). Certains auteurs mettent en avant la capacité de la culture cellulaire d'isoler la souche virale pour permettre par la suite son typage, son sous-typage ou sa caractérisation antigénique complète (important notamment pour la sélection de souches vaccinales du virus influenza) [20]. Même si la RT-PCR ne permet pas de disposer de la souche virale, les extraits obtenus peuvent être congelés, et les produits amplifiés réutilisés par la suite pour une analyse de l'épidémiologie moléculaire, ou une recherche de mutation.

Certains auteurs ont aussi analysé l'aspect économique des RT-PCR multiplex [13,21-23] celui-ci est largement en faveur des techniques moléculaires, surtout pour les techniques « maison ». Au niveau du temps de réalisation, ces techniques durent en moyenne une dizaine d'heures, soit un rendu de résultat au clinicien dans un délai intermédiaire entre l'immunofluorescence (technique la plus rapide) et la culture (technique la plus longue). L'impact clinique de la rapidité des résultats a été étudié par Barenfanger et al. [26]. Cette rapidité s'est traduite par une diminution de la durée moyenne d'hospitalisation et une diminution du coût moyen de séjour. Vingt- quatre heures après le résultat virologique positif, 28,6 \% des prescriptions d'antibiotiques non nécessaires avaient été stoppées au lieu de 9,1\%.

\section{Conclusion}

En conclusion, la plupart des auteurs recommandent pour l'utilisation quotidienne au laboratoire une association de l'immunofluorescence et des techniques moléculaires. L'immunofluorescence permet la communication d'un premier résultat le jour même du prélèvement et reste une bonne technique pour la détection du VRS et de la grippe, les techniques moléculaires améliorent la sensibilité pour les virus conventionnels et permettent d'identifier les virus récemment découverts [21,22,27]. Même si d'importants progrès ont été réalisés dans le diagnostic des maladies virales, établir avec certitude la relation causale entre virus et manifestations cliniques reste encore parfois difficile notamment lors d'immunosuppression thérapeutique. Dans les années à venir, le diagnostic virologique sera d'autant plus important à établir que de nouvelles thérapeutiques antivirales spécifiques seront amenées à être disponibles.

\section{Références}

[1] Gardner PS. Virus infections and respiratory disease of childhood. Arch Dis Child 1968;43:629-45.

[2] Freymuth F, Vabret A, Galateau-Salle F, et al. Detection of respiratory syncytial virus, parainfluenza virus 3 , adenovirus and rhinovirus sequences in respiratory tract of infants by polymerase chain reaction and hybridization. Clin Diagn Virol 1997;8:31-40.

[3] Freymuth F, Vabret A, Galateau F, et al. Etiologie et diagnostic des bronchopneumopathies virales. Ann Biol Clin (Paris) 1998;56:2940.

[4] Steininger C, Kundi M, Aberle SW, et al. Effectiveness of reverse transcription -PCR, virus isolation, and enzyme-linked immunosorbent assay for diagnosis of influenza A virus infection in different age groups. J Clin Microbiol 2002;40:2051-6.

[5] Freymuth F, Vabret A, Rozenberg F, et al. Replication of respiratory viruses, particularly influenza virus, rhinovirus, and coronavirus in HuH7 hepatocarcinoma cell line. J Med Virol 2005;77:295-301.

[6] Freymuth F, Vabret A, Petitjean J, et al. Diagnostic des deux principales viroses respiratoires épidémiques : la grippe et les infections à virus respiratoire syncytial. Place de la virologie moléculaire. Med Mal Infect 2000;30:191-201.

[7] Bellau-Pujol S, Vabret A, Legrand L, et al. Development of three multiplex RT-PCR assays for the detection of 12 respiratory RNA viruses. J Virol Methods 2005;126:53-63.

[8] Vabret A, Gouarin S, Joannes M, et al. Development of a PCR-and hybridization-based assay (PCR Adenovirus Consensus ${ }^{\circledR}$ ) for the detection and the species identification of adenoviruses in respiratory specimens. J Clin Virol 2004;3:116-22.

[9] Freymuth F, Vabret A, Cuvillon-Nimal D, et al. Comparison of multiplex PCR assays and conventional techniques for the diagnostic of respiratory virus infections in children admitted to hospital with an acute respiratory illness. J Med Virol 2006;78:1498-504.

[10] Gilbert LL, Dakhama A, Bone BM, et al. Diagnosis of viral respi- 
ratory tract infections in children by using a reverse transcriptionPCR panel. J Clin Microbiol 1996;34:140-3.

[11] Osiowy C. Direct detection of respiratory syncytial virus, parainfluenza virus, and adenovirus in clinical respiratory specimens by a multiplex reverse transcription-PCR assay. J Clin Microbiol 1998;36:3149-54.

[12] Fan J, Henrickson KJ, Savatski LL. Rapid simultaneous diagnosis of infections with respiratory syncytial viruses A and B, influenza viruses $\mathrm{A}$ and $\mathrm{B}$, and human parainfluenza virus types 1,2 , and 3 by multiplex quantitative reverse transcription-polymerase chain reaction-enzyme hybridization assay (Hexaplex). Clin Infect Dis 1998;26:1397-402.

[13] Hindiyeh M, Hillyard DR, Carroll KC. Evaluation of the Prodesse Hexaplex multiplex PCR assay for direct detection of seven respiratory viruses in clinical specimens. Am J Clin Pathol 2001;116:218-24.

[14] Kehl SC, Henrickson KJ, Hua W, et al. Evaluation of the Hexaplex assay for detection of respiratory viruses in children. J Clin Microbiol 2001;39:1696-701.

[15] Liolios L, Jenney A, Spelman D, et al. Comparison of a multiplex reverse transcription-PCR-enzyme hybridization assay with conventional viral culture and immunofluorescence techniques for the detection of seven viral respiratory pathogens. J Clin Microbiol 2001;39:2779-83.

[16] Henrickson KJ, Hoover S, Kehl KS, et al. National disease burden of respiratory viruses detected in children by polymerase chain reaction. Pediatr Infect Dis J 2004;23:S11-8.

[17] Grondahl B, Puppe W, Hoppe A, et al. Rapid identification of nine microorganisms causing acute respiratory tract infections by singletube multiplex reverse transcription-PCR: feasibility study. J Clin Microbiol 1999;37:1-7.

[18] Puppe W, Weigl JA, Aron G, et al. Evaluation of a multiplex reverse transcriptase PCR ELISA for the detection of nine respiratory tract pathogens. J Clin Virol 2004;30:165-74.
[19] Coiras MT, Perez-Brena P, Garcia ML, et al. Simultaneous detection of influenza A, B, and C viruses, respiratory syncytial virus, and adenoviruses in clinical samples by multiplex reverse transcription nested-PCR assay. J Med Virol 2003;69:132-44.

[20] Weinberg GA, Erdman DD, Edwards KM, et al. Superiority of reverse-transcription polymerase chain reaction to conventional viral culture in the diagnosis of acute respiratory tract infections in children. J Infect Dis 2004;189:706-10.

[21] Rovida F, Percivalle E, Zavattoni M, et al. Monoclonal antibodies versus reverse transcription-PCR for detection of respiratory viruses in a patient population with respiratory tract infections admitted to hospital. J Med Virol 2005;75:336-47

[22] Syrmis MW, Whiley DM, Thomas M, et al. A sensitive, specific, and cost-effective multiplex reverse transcriptase-PCR assay for the detection of seven common respiratory viruses in respiratory samples. J Mol Diagn 2004;6:125-31.

[23] Johnston SL, Sanderson G, Pattemore PK, et al. Use of polymerase chain reaction for diagnosis of picornavirus infection in subjects with and without respiratory symptoms. J Clin Microbiol 1993;31:111-7.

[24] Jartti T, Lehtinen P, Vuorinen T, et al. Persistence of rhinovirus and enterovirus RNA after acute respiratory illnessin children. J Med Virol 2004;72:695-9.

[25] Weigl JA, Puppe W, Grondahl B, et al. Epidemiological investigation of nine respiratory pathogens in hospitalized children in Germany using multiplex reverse-transcriptase polymerase chain reaction. Eur J Clin Microbiol Infect Dis 2000;19:336-43.

[26] Barenfanger J, Drake C, Leon N, et al. Clinical and financial benefits of rapid detection of respiratory viruses: an outcomes study. J Clin Microbiol 2000;38: 2824-8.

[27] Gruteke P, Glas AS, Dierdorp M, et al. Practical implementation of a multiplex PCR for acute respiratory tract infections in children. $\mathrm{J}$ Clin Microbiol 2004;42:5596-603. 